\title{
Loop calculations for lattice matching
}

\section{J.A. Gracey*}

Theoretical Physics Division, Department of Mathematical Sciences, University of Liverpool, P.O. Box 147, Liverpool, L69 3BX, United Kingdom

E-mail: graceyeliv.ac.uk

We report on the recent three loop $\overline{\mathrm{MS}}$ renormalization of the 3-quark operator corresponding to the proton in the chiral limit. The extension of Larin's treatment of $\gamma^{5}$ in dimensional regularization to this case where there is operator mixing is also discussed. The Green's function with the operator inserted at zero momentum at the symmetric point is determined to two loops to aid lattice matching of the same result in the high energy limit.

Loops and Legs in Quantum Field Theory - 11th DESY Workshop on Elementary Particle Physics, April 15-20, 2012

Wernigerode, Germany

\footnotetext{
*Speaker.
} 


\section{Introduction}

The main theoretical formalism used to understand the structure of the particles observed in nature is quantum field theory and in particular Quantum Chromodynamics (QCD). As baryons and mesons are bound states of quarks and gluons, in order to do this one has to calculate in QCD using non-perturbative techniques such as lattice methods which is computationally intense. For instance, to understand proton structure one measures the Green's function of the underlying quantum operator, and its moments, which is built from up, $u$, and down, $d$, quarks. Over recent years there has been interest in assisting lattice measurements of such Green's functions by computing

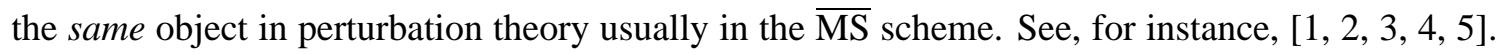
In the high energy limit the lattice results should match on to the perturbative estimates. Indeed in order to reduce errors on the measurements requires perturbative results to as high a loop order as is possible. Currently, with the use of MincER, [6], this is usually three loops. Here we report on a recent computation, [7], involving the 3-quark operator corresponding to the proton. We will present the three loop $\overline{\mathrm{MS}}$ anomalous dimension and note the two loop Green's function with three external quark legs and the operator inserted at zero momentum. The latter will be in an arbitrary linear covariant gauge which will contain the Landau gauge expression the lattice requires. To avoid infrared issues the squared momenta of all three quark legs are equal and non-zero. For this momentum configuration MINCER is not applicable but we will use the Laporta algorithm which in the main is based on integration by parts, [8], in order to determine the Green's function at two loops.

\section{Background}

The particular operators we have renormalized in the $\overline{\mathrm{MS}}$ scheme, [7], are those relating to the proton in the chiral limit which are

$$
\mathscr{O}_{1}=\varepsilon^{I J K} u^{I}\left(\left(u^{J}\right)^{T} C d^{K}\right), \mathscr{O}_{2}=\varepsilon^{I J K} \gamma^{5} u^{I}\left(\left(u^{J}\right)^{T} C \gamma^{5} d^{K}\right)
$$

where $I, J$ and $K$ are the $S U(3)$ colour indices and $C$ is the charge conjugation matrix satisfying

$$
C\left(\gamma^{\mu}\right)^{T} C=\gamma^{\mu}
$$

with $C C=-1$. These operators mix under renormalization and we have carried out two computations. In particular we consider the Green's function $\left\langle\psi_{\alpha}(p) \psi_{\beta}(q) \psi_{\gamma}(-p-q) \mathscr{O}_{i \delta}(0)\right\rangle$ for two different momentum configurations. One is where $q=0$ which allows us to apply the MINCER algorithm to deduce the anomalous dimension mixing matrix to three loops. The other is the case where the external momenta satisfy

$$
p^{2}=q^{2}=r^{2}=-\mu^{2}
$$

where $\mu$ is the mass scale which ensures the coupling constant is dimensionless in $d$ spacetime dimensions. This choice means that no external quark leg is at zero momentum which would be difficult for lattice computations. For the MINCER computation the nullification of one external quark momentum does not introduce infrared infinities since there is always a protecting numerator 
momentum vector from either a quark propagator or a vertex. However, for the full momentum configuration we will only compute to two loops for an arbitrary linear covariant gauge since that is the current range of analytic computation at present. The Landau gauge expression emerges as a corollary. For the $S U(3)$ colour group the operators are gauge invariant and therefore the anomalous dimensions will be independent of the gauge parameter. This will provide a non-trivial internal check on the results. All our computations are carried out using the symbolic manipulation language FORM, [], and its threaded version, [10], including the FORM version of MINCER, [11]. The diagrams are generated with QGRAF, [12]. Overall there are 3 one loop, 40 two loop and 784 three loop Feynman diagrams to be computed.

\section{General Technicalities}

As we are dealing with two operators with the same quantum numbers, 2.1. they will mix under renormalization. This can be easily seen from the structure of $\gamma$-matrices at two loops if one seeds the Green's function with $\mathscr{O}_{1}$. Then the structure $\gamma^{0} \gamma^{1} \gamma^{2} \gamma^{3} \otimes \gamma^{0} \gamma^{1} \gamma^{2} \gamma^{3}$ will be generated with a divergence in $\varepsilon$ where $d=4-2 \varepsilon$. In addition we will have to accommodate $\gamma^{5}$ within the dimensionally regularized calculations. As a result of both of these issues additional operators will be generated under renormalization in $d$ spacetime dimensions, called evanescent operators, which are non-existent in strictly four dimensions. Their effect cannot be omitted from the derivation of the anomalous dimension mixing matrix. The treatment of these three technical problems is related. In general the mixing is handled by a mixing matrix of renormalization constants, $Z_{i j}$, producing an anomalous dimension matrix, $\gamma_{i j}(a)$,

$$
\gamma_{i j}(a)=-\mu \frac{d}{d \mu} \ln Z_{i j}
$$

To treat $\gamma^{5}$ in $d$-dimensions within our automatic symbolic manipulation programmes we have extended the Larin scheme, [13], to the 3-quark operators. Briefly, $\gamma^{5}$ is replaced in $d$-dimensions by a related product of $d$-dimensional $\gamma$-matrices. The renormalization is performed in $d$-dimensions with the renormalization of the evanescent operators being included in an extension of the mixing matrix from two dimensional to infinite dimensional. Though it is a finite matrix order by order in perturbation theory.

To quantify these evanescent operators and to have a full basis for the spinor space in $d$ dimensions we use the generalized $\gamma$-matrices which are defined by, [14, 15],

$$
\Gamma_{(n)}^{\mu_{1} \ldots \mu_{n}}=\frac{1}{n !} \gamma^{\left[\mu_{1}\right.} \ldots \gamma^{\left.\mu_{n}\right]}
$$

For practical programming purposes they can be defined recursively by, [15, 16, 17,

$$
\Gamma_{(n)}^{\mu_{1} \ldots \mu_{n}} \gamma^{v}=\Gamma_{(n+1)}^{\mu_{1} \ldots \mu_{n} v}+\sum_{r=1}^{n}(-1)^{n-r} \eta^{\mu_{r} v} \Gamma_{(n-1)}^{\mu_{1} \ldots \mu_{r-1} \mu_{r+1} \ldots \mu_{n}}
$$

Consequently we have to extend the basis of operators, (2.1), to the $d$-dimensional basis operators,

$$
\begin{aligned}
& \mathscr{O}_{(n)}=\varepsilon^{I J K}\left(\Gamma_{(n)}^{\mu_{1} \ldots \mu_{n}} \psi^{I}\right)\left(\left(\psi^{J}\right)^{T} C \Gamma_{(n) \mu_{1} \ldots \mu_{n}} \psi^{K}\right), n \neq 4 \\
& \mathscr{O}_{(4)}=\frac{\varepsilon^{I J K}}{24}\left(\Gamma_{(4)}^{\mu_{1} \mu_{2} \mu_{3} \mu_{4}} \psi^{I}\right)\left(\left(\psi^{J}\right)^{T} C \Gamma_{(4) \mu_{1} \mu_{2} \mu_{3} \mu_{4}} \psi^{K}\right), n=4
\end{aligned}
$$


where the operator $\mathscr{O}_{2}$ is replaced in $d$-dimensions with $\mathscr{O}_{(4)}$. Though it transpires that under the renormalization only the operators of the form $\mathscr{O}_{(4 n)}$ for positive integer $n$ are generated. As a coding test on the generalized $\gamma$-matrices and the associated FORM module, we have renormalized the diquark operators, [7],

$$
\left.\mathscr{O}_{I_{3} \ldots I_{N_{c}}}^{\mu_{1} \ldots \mu_{n}}\right|_{S U(N c)}=\varepsilon_{I_{1} I_{2} I_{3} \ldots I_{N_{c}}}\left(\psi^{I_{1}}\right)^{T} C \Gamma_{(n)}^{\mu_{1} \ldots \mu_{n}} \psi^{I_{2}}
$$

for the colour group $S U\left(N_{c}\right)$.

To treat $\gamma^{5}$ in $d$-dimensions we first determine what is termed the naive anomalous dimension mixing matrix, $\tilde{\gamma}_{i j}(a)$, where $a=g^{2} /\left(16 \pi^{2}\right)$ and $g$ is the coupling constant. This is derived from the renormalization constants required to make the Green's function finite in $d$-dimensions in the $\overline{\mathrm{MS}}$ scheme. Ignoring the evanescent operator generation problem for the moment $\tilde{\gamma}_{i j}(a)$ would ordinarily be the correct anomalous dimensions. However, as we have computed in $d$-dimensions where there is no concept of chiral symmetry the naive expressions cannot be consistent with an anticommuting $\gamma^{5}$ in strictly four dimensions. To have the correct chiral symmetry, Larin introduced an additional finite renormalization, [13]. Here the corresponding renormalization will be a matrix, $Z_{5, i j}^{\mathrm{fin}}$. It is chosen in such a way that the anticommutativity of $\gamma^{5}$ is restored in strictly four dimensions. In the context of our naive mixing matrix there is therefore a second matrix corresponding to this finite renormalization, $\gamma_{5, i j}(a)$, in order to produce the full anomalous dimensions, $\gamma_{i j}(a)$, via

$$
\gamma_{i j}(a)=\tilde{\gamma}_{i j}(a)+\gamma_{5, i j}(a) \text {. }
$$

The finite renormalization is in essence defined to be the discrepancy in the values of the Green's function with and without the $\gamma^{5}$ after renormalization and in strictly four dimensions. In our case this is formally represented by

$$
\left\langle\mathscr{O}_{1}\right\rangle=Z_{5}^{\mathrm{fin}} \gamma^{5} \otimes \gamma^{5}\left\langle\mathscr{O}_{2}\right\rangle
$$

We have not included the fields with the momentum configuration here as the value of $Z_{5, i j}^{\mathrm{fin}}$ is the same for both calculations which is a strong check on our extension of [13]. A final remark concerns the evanescent operators. Their contribution to the renormalization is to be included in the construction of $\tilde{\gamma}_{i j}(a)$. Then the restoration of chiral symmetry is treated in strictly four dimensions.

\section{Results}

We summarize our results in this section. First, the presence of $\Gamma_{(4)}^{\mu_{1} \mu_{2} \mu_{3} \mu_{4}}$ in the operator $\mathscr{O}_{(4)}$ means that the operator $\mathscr{O}_{(8)}$ will be generated at two loops. This additional operator will, in principle, need to be included in the derivation of the three loop anomalous dimensions. However, using the method of [18] its effect will not appear until four loops as the overlap of $\mathscr{O}_{2}$ with $\mathscr{O}_{(8)}$ does not begin until two loops. The associated anomalous dimension carries two powers of the coupling constant. Next, we have checked that the finite renormalization constant required to restore chiral symmetry in strictly four dimensions is the same for both calculational setups. As in previous finite renormalizations in the $\overline{\mathrm{MS}}$ scheme, [13], the renormalization constant is gauge independent. Although it is a matrix for the proton operator there is no universality in the sense that the constituent elements of the matrix are equivalent to other finite renormalizations associated 
with operators containing $\gamma^{5}$. In other words one has to establish the additional contributions for each individual operator. One expression cannot be translated to another computation with a different operator. Whilst we are computing the three loop anomalous dimensions we only need the finite renormalization to two loops. This is because its conversion to the associated $\gamma^{5}$ anomalous dimension, $\gamma_{5, i j}(a)$ involves the $\beta$-function whose leading term includes the coupling constant. For (2.1) the first row of $\gamma_{5, i j}(a)$ is zero to three loops which is consistent with the $\gamma$-matrix counting. Thus we find

$$
\begin{aligned}
& \gamma_{5,11}(a)=\gamma_{5,12}(a)=O\left(a^{4}\right) \\
& \gamma_{5,21}(a)=125\left[2 N_{f}-33\right] \frac{a^{3}}{27}+O\left(a^{4}\right), \gamma_{5,22}(a)=-500\left[2 N_{f}-33\right] \frac{a^{3}}{27}+O\left(a^{4}\right)
\end{aligned}
$$

as the contribution to the naive anomalous dimension mixing matrix from the finite renormalization where $N_{f}$ is the number of massless quarks.

Thus we find the three loop $\overline{\mathrm{MS}}$ mixing matrix of anomalous dimensions is

$$
\begin{aligned}
\gamma_{11}(a)=\gamma_{22}(a)= & -2 a-\left[2 N_{f}+51\right] \frac{a^{2}}{9} \\
& +\left[260 N_{f}^{2}+[4320 \zeta(3)-4656] N_{f}+1296 \zeta(3)+23481\right] \frac{a^{3}}{162}+O\left(a^{4}\right) \\
\gamma_{12}(a)=\gamma_{21}(a)= & \frac{10}{3} a^{2}+\left[216 \zeta(3)-153-14 N_{f}\right] \frac{a^{3}}{27}+O\left(a^{4}\right)
\end{aligned}
$$

where $\zeta(z)$ is the Riemann zeta function. All four entries in the matrix would be different in the absence of the finite renormalization. At two loops the gauge parameter cancels and our expressions are in agreement with the one and two loop results of [19, 20, 21, 22]. Although we have performed the three loop calculation in the Feynman gauge, the double and triple poles in the three loop renormalization constants are consistent with the renormalization group equation. However, it is the anomalous dimensions of the eigenoperators of (4.2) which are required and we have

$$
\begin{aligned}
\gamma_{+}(a) & =\gamma_{11}(a)+\gamma_{12}(a) \\
& =-2 a-\left[2 N_{f}+21\right] \frac{a^{2}}{9}+\left[260 N_{f}^{2}+[4320 \zeta(3)-4740] N_{f}+2592 \zeta(3)+22563\right] \frac{a^{3}}{162} \\
\gamma_{-}(a) & =\gamma_{11}(a)-\gamma_{12}(a) \\
& =-2 a-\left[2 N_{f}+81\right] \frac{a^{2}}{9}+\left[260 N_{f}^{2}+[4320 \zeta(3)-4572] N_{f}+24399\right] \frac{a^{3}}{162}
\end{aligned}
$$

where the latter corresponds to that of the proton itself.

Finally, in order to do a full lattice matching at two loops we record the Green's function at the symmetric subtraction point. The one loop expression is

$$
\begin{aligned}
\left.\left\langle\psi_{\alpha}(p) \psi_{\beta}(q) \psi_{\gamma}(-p-q) \mathscr{O}_{1 \delta}(0)\right\rangle\right|_{\text {symm }}=\left[1+\frac{2}{9}[\right. & 2 \pi^{2} \alpha+15 \alpha+2 \pi^{2}+15 \\
& \left.\left.-3 \psi^{\prime}\left(\frac{1}{3}\right)-3 \psi^{\prime}\left(\frac{1}{3}\right) \alpha\right] a\right] I_{\alpha \delta} \otimes I_{\beta \gamma} \\
+ & {\left[3 \psi^{\prime}\left(\frac{1}{3}\right) \alpha+3 \psi^{\prime}\left(\frac{1}{3}\right) \alpha-2 \pi^{2} \alpha-2 \pi^{2}\right] } \\
\times & {\left[4 I_{\alpha \delta} \otimes \sigma_{\beta \gamma}^{p q}+2 \sigma_{\alpha \delta}^{p \mu} \otimes \sigma_{p \mu \beta \gamma}\right.} \\
& \left.+4 \sigma_{\alpha \delta}^{p \mu} \otimes \sigma_{q \mu \beta \gamma}\right] \frac{a}{81 \mu^{2}}+O\left(a^{2}\right)
\end{aligned}
$$


for arbitrary linear covariant gauge where $\psi(z)$ is the derivative of the logarithm of the Euler $\Gamma$ function. The two loop expression is too cumbersome to record here but can be found in [7. Though we note that the computations for this momentum configuration used the REDUZE version, [23], of the Laporta algorithm, [8], and is based in GiNAC, [24]. At two loops the masters involve conventional and harmonic polylogarithms, [25, 26, 27, 28], and are summarized in [5]. The expression for the operator $\mathscr{O}_{2}$ can be deduced from (4.4) by multiplying by $\gamma^{5} \otimes \gamma^{5}$ and respecting (2.2).

\section{Discussion}

We have presented an overview of the current state of play with regard to computing the renormalization of 3-quark operators to three loops in the $\overline{\mathrm{MS}}$ scheme. In addition we have determined the appropriate Green's functions to two loops in order to aid lattice matching of the same quantity. In terms of spin- $(j, \bar{j})$ representations of the Lorentz group our results correspond to an operator in the $D_{\left(\frac{1}{2}, 0\right)}$ representation of both chiralities. There are several future directions with this analysis. One is to extend to other baryons and representations such as $D_{\left(1, \frac{1}{2}\right)}$ and $D_{\left(\frac{3}{2}, 0\right)}$. Equally in order to refine proton structure measurements further, knowledge of the renormalization of higher moments of the operators for moments $n \leq 2$, are required. For example, this would be relevant to the study of distribution amplitudes.

\section{References}

[1] K.G. Chetyrkin \& A. Rétey, Renormalization and running of quark mass and field in the regularization invariant and $\overline{M S}$ schemes at three loops and four loops, Nucl. Phys. B583 (2000) 3 [hep-ph/9910332].

[2] J.A. Gracey, Three loop anomalous dimension of nonsinglet quark currents in the RI' scheme, Nucl. Phys. B662 (2003) 247 [hep-ph/ 0304113$].$

[3] C. Sturm, Y. Aoki, N.H. Christ, T. Izubuchi, C.T.C. Sachrajda \& A. Soni, Renormalization of quark bilinear operators in a momentum-subtraction scheme with a nonexceptional subtraction point, Phys. Rev. D80 (2009) 014501 [arXiv: 0901.2599 ].

[4] M. Gorbahn \& S. Jäger, Precise $\overline{M S}$ light-quark masses from lattice QCD in the RI/SMOM scheme, Phys. Rev. D82 (2010) 114001 [arXiv: 1004 . 3997].

[5] L.G. Almeida \& C. Sturm, Two-loop matching factors for light quark masses and three-loop mass anomalous dimensions in the RI/SMOM schemes, Phys. Rev. D82 (2010) 054017 [arXiv:1004.4613].

[6] S.G. Gorishny, S.A. Larin, L.R. Surguladze \& F.K. Tkachov, Mincer: program for multiloop calculations in quantum field theory for the Schoonschip system, Comput. Phys. Commun. 55 (1989) 381.

[7] J.A. Gracey, paper in preparation (2012).

[8] S. Laporta, High precision calculation of multiloop Feynman integrals by difference equations, Int. J. Mod. Phys. A15 (2000) 5087 [hep-th/ 0207004 ].

[9] J.A.M. Vermaseren, New features of FORM, math-ph/0010025 [math-ph/0010025]. 
[10] M. Tentyukov \& J.A.M. Vermaseren, The multithreaded version of FORM, Comput. Phys. Commun. 181 (2010) 1419 [hep-ph/ 0702279$].$

[11] S.A. Larin, F.V. Tkachov \& J.A.M. Vermaseren, The Form version of Mincer, NIKHEF-H-91-18.

[12] P. Nogueira, Automatic Feynman graph generation, J. Comput. Phys. 105 (1993) 279.

[13] S.A. Larin, The renormalization of the axial anomaly in dimensional regularization, Phys. Lett. $\mathbf{B 3 0 3}$ (1993) 113 [hep-ph/9302240].

[14] A.D. Kennedy, Clifford algebras in $2 \omega$ dimensions, J. Math. Phys. 22 (1981) 1330.

[15] A.N. Vasil'ev, S.É. Derkachov \& N.A. Kivel, A technique for calculating the $\gamma$-matrix structures of the diagrams of a total four fermion interaction with infinite number of vertices in $d=(2+\varepsilon)$-dimensional regularization, Theor. Math. Phys. 103 (1995) 487.

[16] A.N. Vasil'ev, M.I. Vyazovskii, S.É. Derkachov \& N.A. Kivel, On the equivalence of renormalizations in standard and dimensional regularizations of 2-D four-fermion interactions, Theor. Math. Phys. 107 (1996) 441.

[17] A.N. Vasil'ev, M.I. Vyazovskii, S.É. Derkachov \& N.A. Kivel, Three-loop calculation of the anomalous field dimension in the full four-fermion $U(N)$-symmetric model, Theor. Math. Phys. 107 (1996) 710.

[18] A. Bondi, G. Curci, G. Paffuti \& P. Rossi, Metric and central charge in the perturbative approach to two-dimensional fermionic models, Annals Phys. 199 (1990) 268.

[19] G.P. Lepage \& S.J. Brodsky, Exclusive processes in quantum chromodynamics: the form-factors of baryons at large momentum transfer, Phys. Rev. Lett. 43 (1979) 545.

[20] G.P. Lepage \& S.J. Brodsky, Exclusive processes in perturbative quantum chromodynamics, Phys. Rev. D22 (1980) 2157.

[21] M.E. Peskin, Anomalous dimensions of three quark operators, Phys. Lett. B88 (1979) 128.

[22] A.A. Pivovarov \& L.R. Surguladze, Anomalous dimensions of octet baryonic currents in two loop approximation, Nucl. Phys. B360 (1991) 97.

[23] C. Studerus, Reduze - Feynman integral reduction in C++, Comput. Phys. Commun. 181 (2010) 1293 [arXiv:0912.2546].

[24] C.W. Bauer, A. Frink \& R. Kreckel, Introduction to the GiNaC framework for symbolic computation within the $C++$ programming language, cs/0004015 [cs/ 0004015 ].

[25] A.I. Davydychev, Recursive algorithm of evaluating vertex type Feynman integrals, J. Phys. A25 (1992) 5587.

[26] N.I. Usyukina \& A.I. Davydychev, Some exact results for two loop diagrams with three and four external lines, Phys. Atom. Nucl. 56 (1993) 1553 [hep-ph/9307327].

[27] N.I. Usyukina \& A.I. Davydychev, New results for two loop off-shell three point diagrams, Phys. Lett. B332 (1994) 159 [hep-ph/9402223].

[28] T.G. Birthwright, E.W.N. Glover \& P. Marquard, Master integrals for massless two-loop vertex diagrams with three offshell legs, JHEP 0409 (2004) 042 [hep-ph / 0407343 ]. 